\title{
Limited Predictability of the Future Thermohaline Circulation Close to an Instability Threshold
}

\author{
Reto Knutti And Thomas F. Stocker \\ Climate and Environmental Physics, Physics Institute, University of Bern, Bern, Switzerland
}

(Manuscript received 19 April 2001, in final form 30 July 2001)

ABSTRACT

\begin{abstract}
Most ocean-atmosphere models predict a reduction of the thermohaline circulation for a warmer climate in the near future. Although a reduction in the Atlantic Ocean circulation appears to be a robust result, the question remains open whether the climate system could possibly cross a critical threshold leading to a complete shutdown of the North Atlantic deep-water formation. Ensemble simulations with an ocean-atmosphere climate model of reduced complexity are performed to investigate the range of possible future climate evolutions when the climate system is close to such a threshold. It is found that the sensitivity of the ocean circulation to perturbations increases rapidly when approaching the bifurcation point, thereby severely limiting the predictability of future climate. At the bifurcation point, different response types such as linear responses, nonlinear transitions, or resonance behavior are observed. Close to the threshold, thermohaline shutdowns can occur thousands of years after the warming has stopped. A characterization of the probability for the different response types reveals a more complex picture for the future evolution of the ocean circulation than previously assumed. These results raise fundamental questions of how far the large differences in projections of the Atlantic circulation response to global warming are caused by different representations of processes, parameterizations, and/or resolution in individual models and whether the predictability of the Atlantic circulation becomes inherently limited when approaching a bifurcation point.
\end{abstract}

\section{Introduction}

Anticipated future warming of the climate system has the potential to weaken the thermohaline circulation (THC) by reducing surface water density in the formation regions of North Atlantic Deep Water (NADW) through both high-latitude warming and enhanced poleward moisture transport of the atmosphere (Manabe and Stouffer 1993; Houghton et al. 2001). An important related issue is the question whether the climate system could possibly cross a critical threshold leading to a nonlinear transition of the THC to a qualitatively different circulation mode without deep-water formation in the North Atlantic. Such a reorganization would have a profound impact on the climate of western and central Europe, on ocean biogeochemical cycles (Joos et al. 1999), and future sea level (Knutti and Stocker 2000). Of special concern is the possibility that such reorganizations may be irreversible due to the existence of multiple equilibria and hysteresis behavior in the climate system (Stocker and Wright 1991a; Mikolajewicz and Maier-Reimer 1994; Rahmstorf 1995). For present-day conditions, multiple equilibria of the thermohaline cir-

Corresponding author address: Reto Knutti, Climate and Environmental Physics, Physics Institute, University of Bern, Sidlerstrase 5, CH-3012 Bern, Switzerland.

E-mail: knutti@climate.unibe.ch culation have been found in the entire hierarchy of ocean and climate models (Stommel 1961; Stocker and Wright 1991a; Manabe and Stouffer 1988).

Recent model simulations show, that substantial changes in the Atlantic THC may occur spontaneously in the coupled atmosphere-ocean system, even when no external forcing is applied. Hall and Stouffer (2001) found in their 15000 -yr control simulation with a comprehensive coupled climate model a cooling event that was associated with a reduction of the THC for a few decades. The event was triggered by persistent anomalies of the atmospheric circulation over the North Atlantic, that is, short-term fluctuations of atmosphereocean fluxes (and their integrated effects) are able to initiate longer-term changes in the THC. A study with a simplified coupled model similarly suggests that random atmospheric forcing may lead to large excursions in the THC strength of the Atlantic (Aeberhardt et al. 2000). Changes in the THC thus contribute to natural variability in the climate system.

The Atlantic THC may also respond directly to perturbations in the forcing. Under global warming, most comprehensive ocean-atmosphere models show a significant reduction of NADW formation (see Houghton et al. 2001 and refs. therein). For strong warming, different models simulate a complete shutdown of the THC (Manabe and Stouffer 1993; Stocker and Schmittner 
1997). However, recent studies also suggest that the THC may be stabilized through increased wind-driven evaporation (Gent 2001) and trends in the North Atlantic oscillation (Delworth and Dixon 2000) or the El NiñoSouthern Oscillation (Latif et al. 2000). The stability of the THC in climate models depends on many factors, among them the response of the hydrological cycle to the warming, the parameterization of mixing processes in the ocean (Knutti et al. 2000), the rate of greenhouse gas increase (Stocker and Schmittner 1997), and on the initial strength of the THC itself (Tziperman 2000). The Intergovernmental Panel on Climate Change (IPCC) has analyzed the most recent model projections and concludes in their Third Assessment Report that a reduction of the Atlantic THC is a likely response to increased greenhouse gas forcing, but that during this century, a complete shutdown is less likely than previously assumed. However, if the rate of change in radiative forcing remains large and lasts long enough, a complete shutdown after the year 2100 cannot be excluded.

The purpose of this paper is to investigate the limitation of predictability of the THC on climatic timescales when the system has more than one stable equilibrium. The possibility of changes in the Atlantic THC in response to global warming reduces predictability not only if a transition occurs but deteriorates predictability increasingly as the transition threshold is approached. In a nonlinear system certain variables can be predicted only in a probabilistic sense. This implies that multimodel, multi-initial condition simulations must be performed in order to determine the probability distribution of these variables and their changes due to external forcing (Palmer 1999). This approach is now routine for long-term weather forecast, but only few studies address this topic on climatic timescales (e.g., Stott et al. 2000).

The paper is organized as follows. Section 2 describes the model and explains the random forcing. Ensemble simulations are presented in section 3 for cases below and at the instability threshold, sensitivities are investigated, and probabilities of a THC shutdown are determined as a function of climate sensitivity and forcing amplitude. Conclusions are given in section 4 .

\section{Model description}

We use a climate model of reduced complexity to explore the range of possible future evolutions of the THC when approaching the model's threshold for a shutdown of NADW formation. The model consists of a zonally averaged ocean model resolving individually the Pacific, Atlantic, Indian, and Southern Oceans (Stocker and Wright 1991b), coupled to a zonally and vertically averaged energy-balance atmosphere (Stocker et al. 1992) with an active hydrological cycle (Schmittner and Stocker 1999). The latitudinal resolution is set to $5^{\circ}$, increased compared to earlier versions (Knutti and Stocker 2000), and the seasonal cycle (Schmittner and Stocker 2001) is resolved. The simple geographical structure restricts studies to scales of thousands of kilometers, on which results are generally consistent with those of comprehensive 3D models. However, while fully coupled atmosphere-ocean general circulation models (AOGCMs) base their conclusions on just a few model runs due to computational constraints, the efficiency of the model allows us to run thousands of simulations. This opens the field for a new approach: ensemble simulations and the use of statistical methods to gain new insight into the future of the thermohaline circulation.

The model has a rather constant annual mean steady state for modern boundary conditions, mainly due to the lack of dynamics in the atmosphere. The climate system, however, is characterized by natural variability that produces states that are only statistically steady. Atmospheric weather processes occur on much shorter timescales than ocean processes of similar spatial extent. The ocean acts as an integrator of this short-period forcing, and describing it as stochastic perturbations is a good first approximation (Hasselmann 1976; Frankignoul and Hasselmann 1977). From the large-scale ocean perspective, we can thus obtain important insight into the dynamical behavior and variability of the ocean circulation by including this stochastic forcing process into the model. For the ensemble simulations we artificially prescribe atmospheric variability by applying small freshwater fluxes at the ocean surface. The random freshwater forcing consists of spatially uncorrelated Gaussian white noise applied to every ocean surface cell with a standard deviation $\Delta F$ ranging from zero to $0.07 \mathrm{~Sv}\left(1 \mathrm{Sverdrup}=10^{6} \mathrm{~m}^{3} \mathrm{~s}^{-1}\right)$. The total net freshwater forcing is zero at every time step to avoid climate drift. The results reported here are obtained by starting from the same initial steady state and calculating one hundred identical transient warming simulations. The forcing standard deviation $\Delta F$ is constant in time and identical for the one hundred simulations, the only difference are the random number sequences. Each model run will thus project the future climate evolution for the chosen warming scenario, yet the projections will slightly differ due to the applied variability. An other method to introduce uncertainty into the model results that is actually used in weather forecasting is to slightly change boundary or initial conditions for every model run, but the applied perturbation would be difficult to quantify. For the surface freshwater forcing, we can relate our results to a single number, the standard deviation of the applied freshwater noise.

For the simulation of global warming, we first apply the stochastic forcing to constant modern climate during $1000 \mathrm{yr}$ to let the model adjust to the noise, then we double atmospheric $\mathrm{CO}_{2}$ with an increase rate of $0.5 \%$ $\mathrm{yr}^{-1}$. The atmospheric $\mathrm{CO}_{2}$ concentration thus reaches 560 ppmv after $140 \mathrm{yr}$ and is kept constant afterward. The additional radiative forcing at the top of the atmosphere consists of a term depending logarithmically on the $\mathrm{CO}_{2}$ concentration and an additive feedback term, 

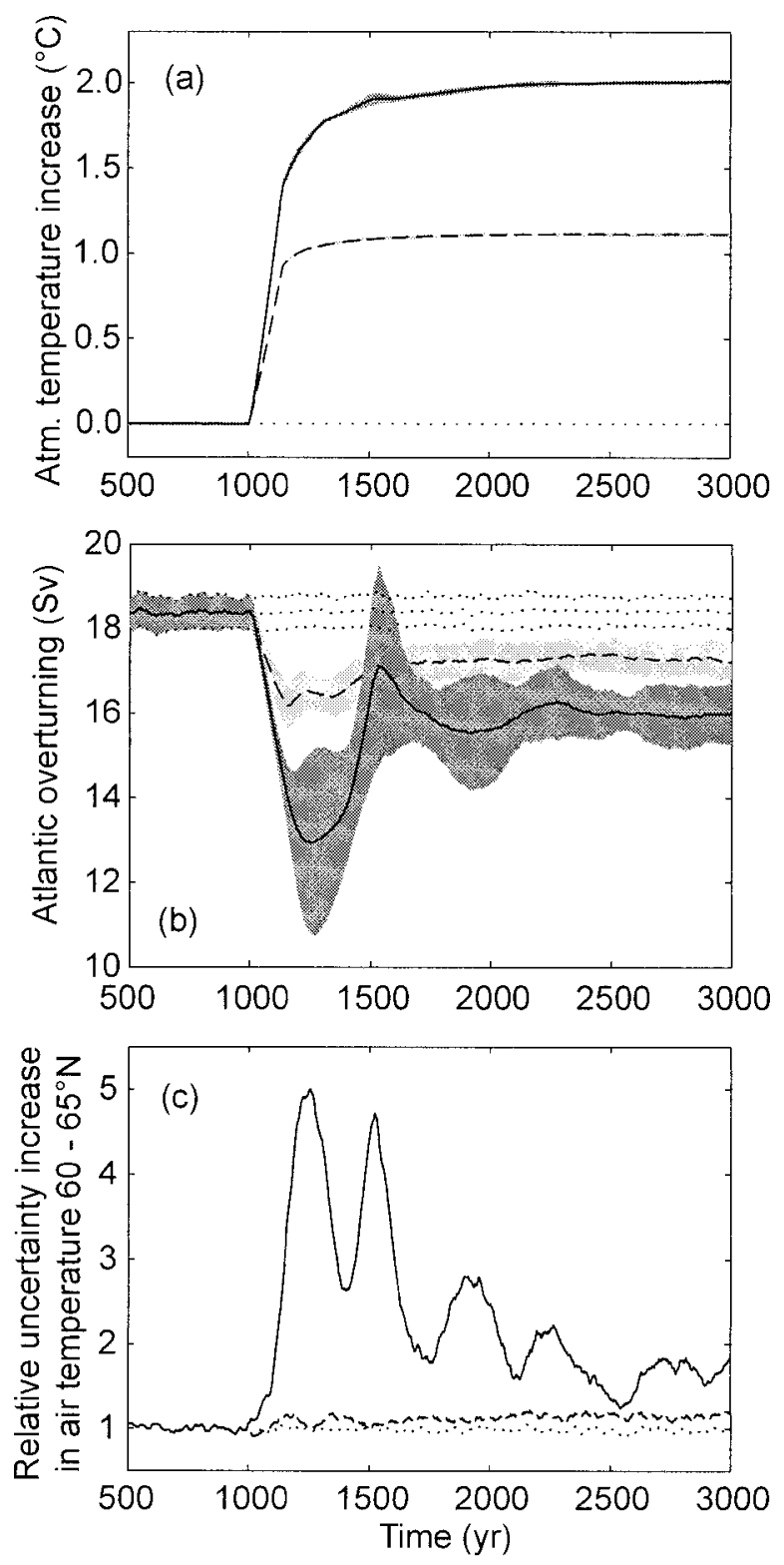

FIG. 1. (a) Globally averaged surface air temperature and (b) Atlantic overturning in a $2 \times \mathrm{CO}_{2}(140 \mathrm{yr})$ scenario for a weak (dashed) and a strong (solid) global warming ensemble set, plus a control set (dotted). The mean and std dev for 100 ensemble runs are shown. When the ocean-atmosphere system approaches the point of a possible THC collapse (dark shading) during the transient phase, small perturbations can strongly affect the response of the thermohaline circulation, thereby severely limiting the predictability of the future THC evolution. (c) Shows the resulting uncertainty (ensemble std dev) in the projected surface air temperature at $60^{\circ}-65^{\circ} \mathrm{N}$, relative to 100 control runs without any warming, indicating that the uncertainty in climatic variables associated with the thermohaline circulation may increase by a factor of 5 just by approaching (but not crossing) the critical threshold.

which depends linearly on the atmospheric temperature increase. This feedback term mimics the indirect effect of changes in atmospheric water vapor and can be adjusted to obtain different climate sensitivities (global mean atmospheric temperature increase for doubling $\mathrm{CO}_{2}$ ). However, we note that our main qualitative conclusions neither depend on the parameterization of the radiative forcing nor on the applied $\mathrm{CO}_{2}$ scenario.

\section{Evolutions of the THC in ensemble simulations \\ a. Behavior below the instability threshold}

The IPCC concludes in its Third Assessment Report that a shutdown of the thermohaline circulation in the next $100 \mathrm{yr}$ is unlikely. This may imply that there is less cause for concern about future changes of the thermohaline circulation than previously assumed. Here we show that such an interpretation would be wrong. The thermohaline circulation becomes very sensitive to small perturbations simply by approaching but not crossing the critical threshold for a collapse. To illustrate this effect, we calculated two sets of global warming ensemble simulations, one with a weak atmospheric warming of about $1{ }^{\circ} \mathrm{C}$ (Fig. 1a, dashed) and a second with a stronger warming of nearly $2^{\circ} \mathrm{C}$ (Fig. 1a, solid). For the latter, the climate sensitivity was chosen at the highest value where none of the individual runs predicts a shutdown of NADW formation. For both ensemble sets, 100 simulations with stochastic forcing $\Delta F=0.05$ $\mathrm{Sv}$ were performed and the mean and uncertainty of the model projections are plotted as a line and a shaded band, respectively. Here we define the uncertainty of the ensemble model projection at a given time as the standard deviation of the 100 individual projections of the ensemble at that time. Control runs without warming are shown for comparison (dotted line and band). The uncertainty in global mean air temperature due to the stochastic forcing is small (Fig. 1a), because the fluctuations mainly affect the transport and distribution of heat, but not its global mean. While the uncertainty in the projected strength of the thermohaline circulation remains almost constant for the weak warming scenario (Fig. 1b, width of the light shaded band), the individual model projections disagree for the strong warming scenario, resulting in a large uncertainty of the THC reduction during the transient phase (Fig. 1b, dark shaded band). This is directly translated into a large uncertainty in the Northern Hemisphere climate. For example, the range of the projected atmospheric temperature between $60^{\circ}$ and $65^{\circ} \mathrm{N}$ increases by up to a factor of 5, relative to the control runs (Fig. 1c). For smaller areas around the North Atlantic region, the uncertainty increase could even be larger, but comprehensive models with higher resolution are necessary to quantify the effects on smaller scales. We conclude from these simulations that the thermohaline circulation will behave in a way that the predictability is inherently limited when approaching the region of a phase transition.

\section{b. THC bifurcations at the instability threshold}

Next we increase the climate sensitivity $\Delta T_{2 \times}$ such that for a given perturbation amplitude $\Delta F$ the model 

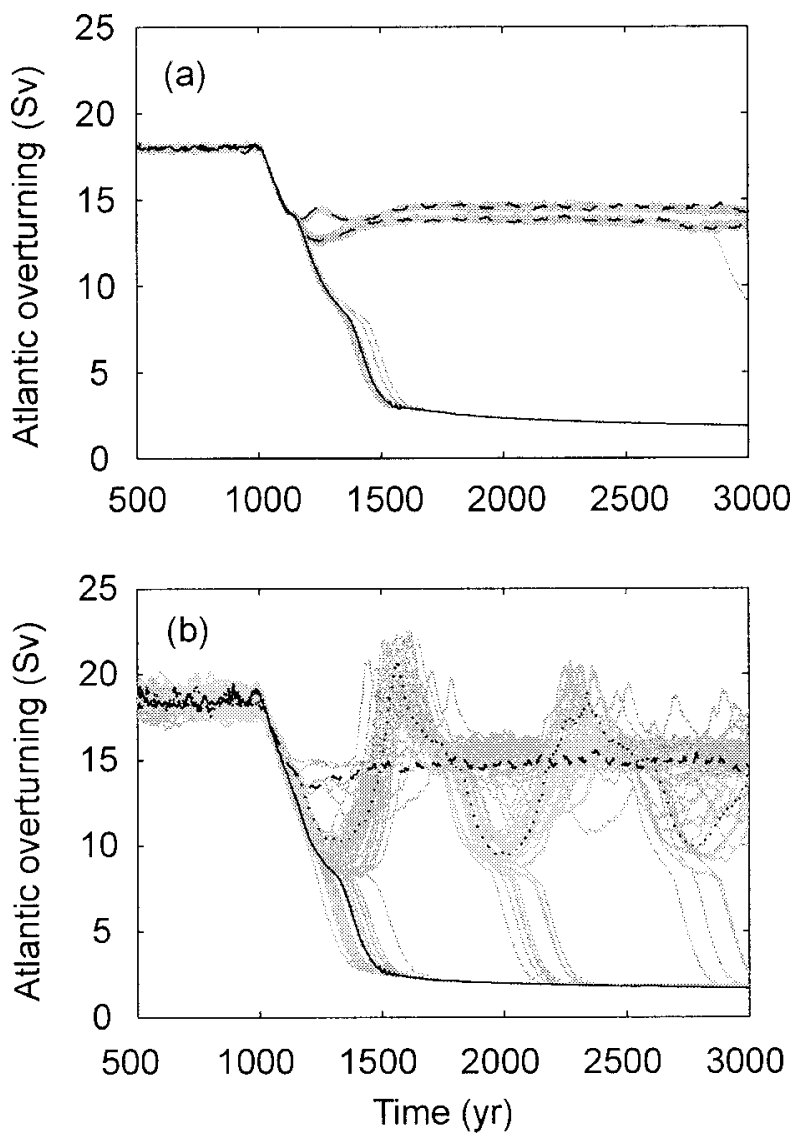

FIG. 2. Evolution of the Atlantic overturning for 100 simulations using stochastic forcing (a) $\Delta F=0.02 \mathrm{~Sv}$ and (b) $\Delta F=0.05 \mathrm{~Sv}$ in the critical range for a THC collapse. All ensemble members are plotted as gray lines, and some projections are highlighted in black for clarity. At least in this model, the possible evolutions seem to gather in clusters of reduced active overturning (dashed), collapsed overturning (solid), and resonance-like behavior (dotted).

reaches the instability threshold (the relation of $\Delta F$ and $\Delta T_{2 \times}$ is discussed in section $3 \mathrm{e}$ ), that is, some of the simulations in the ensemble exhibit a THC shutdown, while others show a weakening and recovering circulation. This is shown in Fig. 2a for a small stochastic forcing $(\Delta F=0.02 \mathrm{~Sv})$ and in Fig. $2 \mathrm{~b}$ for $\Delta F=0.05$ $\mathrm{Sv}$ as in Fig. 1. Note that again the global warming scenario and the stochastic forcing amplitude $\Delta F$ are identical for all 100 simulations in each ensemble set. All ensemble members are plotted as gray lines, and some projections are highlighted in black for clarity. A few remarkable conclusions are evident from Fig. 2. First, even for small noise applied at the surface, certain global warming scenarios exist where only a probabilistic prediction of the final state is possible. The applied noise in Fig. 2a causes THC variations in the steady states that are much smaller than the THC variability in $3 \mathrm{D}$ atmosphere-ocean models. Our findings are therefore not due to unrealistically strong perturbations. Second, while the specific response of the THC certainly depends on the model type, the model parameters and the forcing, the different evolutions of the THC always clearly gather into clusters. There seems to be no continuous range but only a limited number of preferred future evolutions. Third, for a once reduced active THC, more than one evolution and new equilibrium state seem to be possible (Fig. 2a, dashed lines), and no transition between the two active states is observed. The two new steady states are very similar and differ only by their convection pattern and deep-water formation rate in the North Atlantic. This issue has never been addressed before, and at the moment we cannot decide whether this may be true for the natural climate system or whether this is a model-specific result. However, this would certainly be an important point to investigate using more complex, comprehensive models. Fourth, besides the rather linear response of a recovering (dashed) and the nonlinear response of a collapsing THC (solid), which appear also for weak stochastic forcing (Fig. 2a), a resonance-like behavior (dotted) is found for stronger stochastic forcing (Fig. 2b), at least in this model configuration. Similar century-timescale variations have been observed in previous studies using earlier versions of this model (Aeberhardt et al. 2000) and were interpreted to be resonance frequencies of the ocean-atmosphere model, which are parameter and model dependent but rather independent of the forcing timescale. Another possible interpretation of these oscillations is that the ocean has been forced outside the stable regime (Tziperman 1997, 2000).

The question arises whether the oscillations shown in Fig. $2 b$ are robust or not. One might conclude a number of facts from Fig. 2; namely, that oscillations only appear for high perturbation amplitudes, that their amplitude is decreasing with time, or that oscillations will not reappear if once sufficiently damped. It is also tempting to interpret the timescale of these oscillations with regard to the rapid, almost periodic climate changes during the last ice age, suggesting that the climate system was closer to a threshold than today. Figure 3 shows the ensemble sets around the bifurcation point for the same perturbation amplitudes as in Fig. 2, but for an earlier model version without seasonal cycle and with coarser resolution (Knutti and Stocker 2000). The three main types (black lines) largely remain, and the clustering into preferred solutions also seems robust. With regard to the appearance, the timescale and amplitude of the oscillations, however, neither of the hypothesized conclusions above is justified. While the underlying processes are certainly worth investigating, a detailed interpretation of the climatic relevance of these oscillations would be inappropriate at this stage.

In agreement with the studies of Tziperman (1997), the present results show that all possible evolutions of the THC end up in a collapsed state or a stable active circulation of at least $12 \mathrm{~Sv}$; there is no intermediate state with weak (less than about $12 \mathrm{~Sv}$ ) overturning in the Atlantic in this model. 

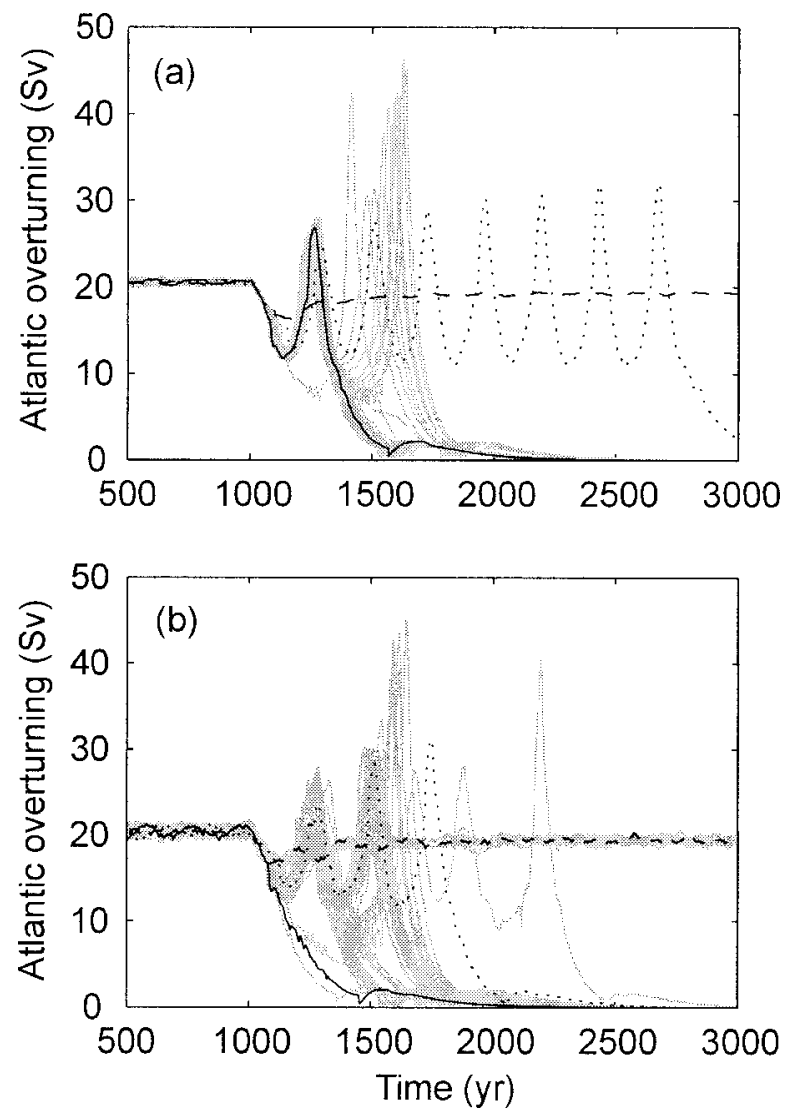

FIG. 3. Same as Fig. 2, but for an earlier model version (Knutti and Stocker 2000). While the three main response types (black lines) are robust, the characteristics of the oscillations are strongly parameter and model dependent.

\section{c. Long-term evolution of the THC}

To gain further insight into the long-term behavior of the THC close to the instability threshold, the ensembles shown in Fig. 2 were integrated for another 8000 yr. The two long ensemble sets are shown in Fig. 4, with two arbitrarily chosen ensemble members highlighted as black lines. While the active steady states in Fig. 4 a are quickly approached, there is still a very slow adjustment to equilibrium that is sufficient in some cases to cause a sudden breakdown of the THC thousands of years after the warming has stopped. Note that the random perturbations themselves cause no drift in the globally averaged salinity. While such small changes would not even be detectable in short integrations, they are large enough in this case to shift the model into a new circulation state. One reason for this is, of course, that the climate sensitivity is intentionally chosen to exactly reach the bifurcation point. While many spontaneous collapses from the lower active branch are observed (Fig. 4a, e.g., solid black line), only three members of the upper branch (dashed black line) collapse. No transition between the two active branches is observed. The solid black line in Fig. 4b further shows that the oscil- lations may not necessarily be continuous, but can vanish and reappear. However, they never reach the initial amplitude induced by the global warming. A key point that we would like to emphasize is that transitions from an active to a collapsed circulation state can occur long after the initial perturbation (i.e., here the global warming) has stopped, if the system is close enough to the instability threshold.

One might argue that the Gaussian distribution of the applied stochastic noise includes a small but nonzero probability for very large freshwater perturbations in the convection regions of the deep-water formation, having the potential to cause the spontaneous late THC collapses. However, additional simulations with the Gaussian distribution limited to two standard deviations yielded very similar results and did not prevent the spontaneous late collapses shown in Fig. 4. Also, from Fig. 4 one might conclude that the collapses are extremely sudden. But a detailed view reveals that the strength of the THC reduces over several decades by a few percent before shutting down abruptly, supporting the hypothesis that the collapse develops through internal dynamics of the climate system.

\section{d. Sensitivity to stochastic forcing}

In Fig. 1, we have illustrated that the uncertainty in the projections strongly increases when the system approaches an instability threshold. Next we show (Fig. 5) that the variability, that is, the sensitivity of some variables to the stochastic forcing in each single model run increases as well during the warming. To estimate the variability, we subtract the 20 -yr running mean from the time series of surface air temperature at $60^{\circ}-65^{\circ} \mathrm{N}$ to remove warming trends. Then we calculate standard deviations in a 20-yr moving window and average these results over all 100 runs of the ensemble. Figure 5 shows the increase in variability relative to the control average for the weak (dashed) and strong (solid) warming scenario already discussed in Fig. 1. The control runs are shown for comparison (dotted). In this case, the variability imposed by the stochastic forcing increases by up to $25 \%$. The variability increase is larger in the Atlantic than in the other basins and largest in the North Atlantic region. A recent study with a comprehensive 3D ocean-atmosphere model also suggests a variability increase close to the instability threshold (Tziperman 2000). We note that in contrast to the projection uncertainty that decreases again in the new steady state (Fig. 1c), the increased variability remains at a constant high level (Fig. 5).

\section{e. The probabilistic view}

Combining these findings, we can draw a qualitative picture for a shutdown of the thermohaline circulation by relating it to the applied stochastic forcing (mimicking natural variability and noise through stochastic pro- 

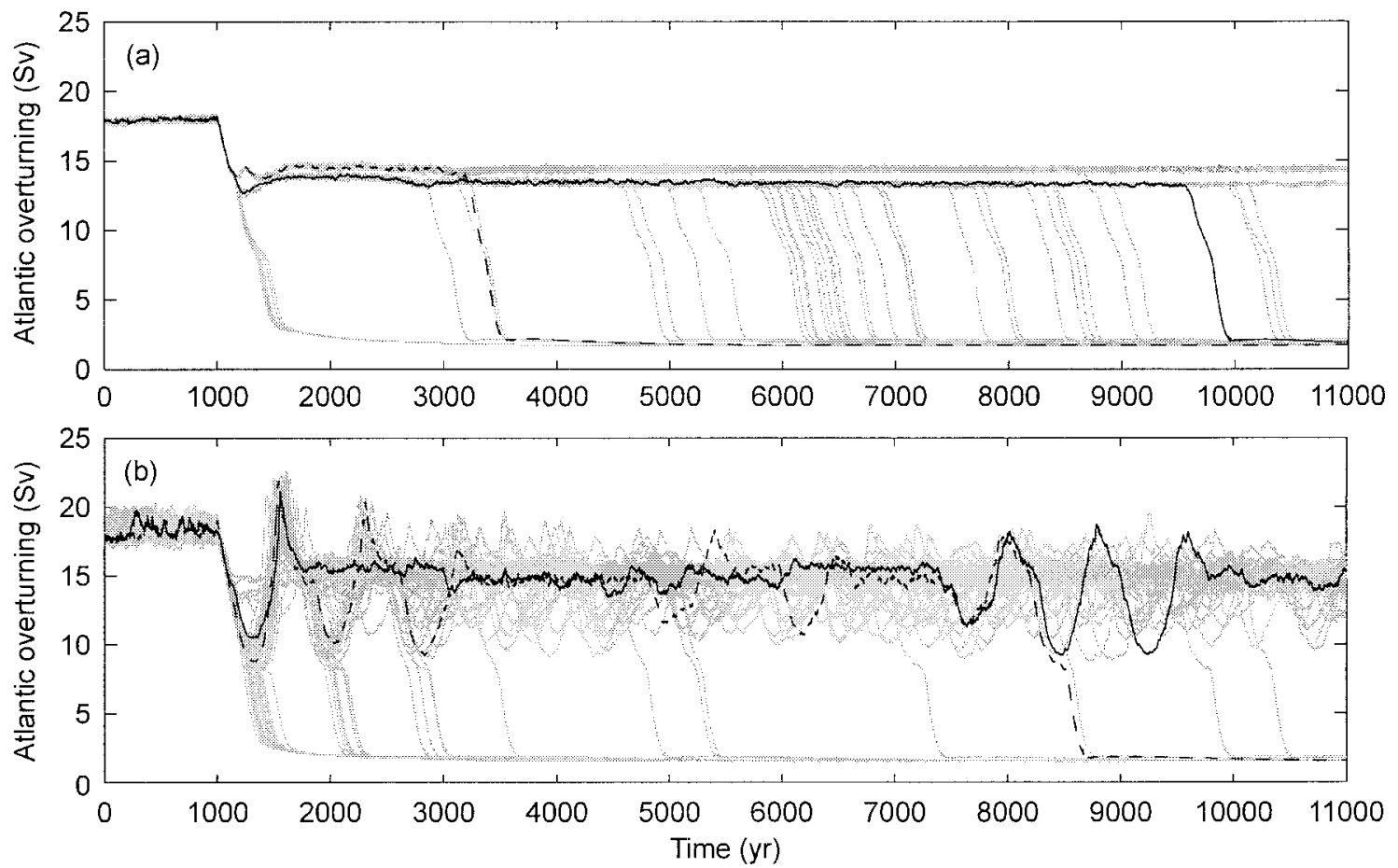

FIG. 4. Expansion of Fig. 2 to 11000 yr. Spontaneous shutdowns of the thermohaline circulation may occur long after the warming has stopped, if the system is close to the instability threshold.

cesses like weather) and to the climate sensitivity, that is, the strength of the projected warming. This is shown in Fig. 6a. The contour lines are interpolated from 100 simulations calculated at each point in the parameter space indicated by a dot and denote the percentage of cases for which the THC is in the collapsed state 1000 years after the start of the warming. For low climate sensitivities (measured as global mean air temperature increase for doubling atmospheric $\mathrm{CO}_{2}$ ), the THC remains active in all model simulations, whereas it col-

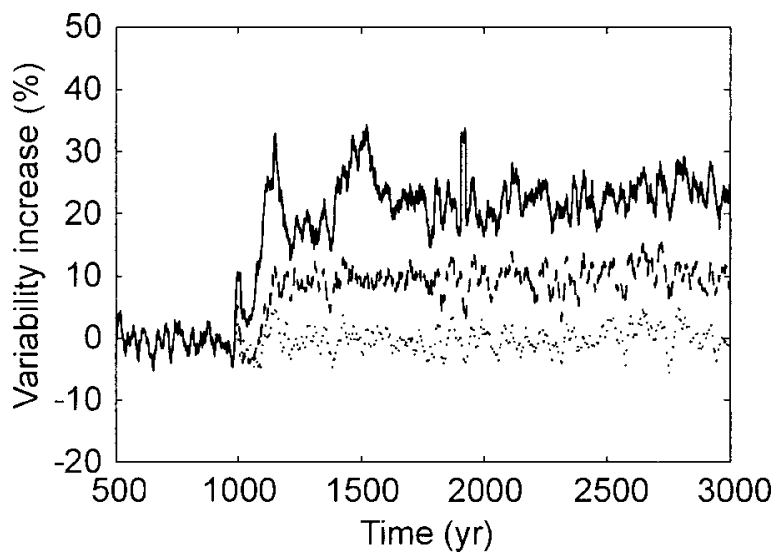

FIG. 5. Variability increase in projected surface air temperature at $60^{\circ}-65^{\circ} \mathrm{N}$ for the weak (dashed) and strong (solid) warming scenarios shown in Fig. 1, relative to the control simulations (dotted). The variability increases by up to $25 \%$ when the warming starts and persists after the system has come to a new equilibrium. lapses for high climate sensitivities. However, for nonzero stochastic forcing a considerably broad range of climate sensitivities exists around the bifurcation point where both, a recovering or a collapsing circulation is possible. This range of climate sensitivities widens and shifts toward lower values for increasing $\Delta F$. The latter is obvious, since both stronger warming and stronger stochastic forcing shift the system toward the instability threshold. A surprising feature is the separation into two seemingly different regimes for weak or strong stochastic forcing. For a given probability contour line, the climate sensitivity depends linearly on the freshwater forcing amplitude only above about $0.04 \mathrm{~Sv}$, but not below. At the moment, we have no hypothesis for this effect.

A shutdown of the thermohaline circulation is possible long after the $\mathrm{CO}_{2}$ concentration has stabilized at a new level (Fig. 4). Figure 6b indicates that at least for stronger stochastic forcing and a given climate sensitivity, there may be a substantial probability (up to $25 \%$ ) for a shutdown of the THC between year 1000 and 2000 after the start of the warming. This means that it may not be sufficient to integrate fully coupled AOGCMs only until the thermohaline circulation starts to recover again. Although computationally expensive, integrations over many centuries or even millennia are needed to determine whether the THC will collapse or not, if there is reason to believe that the model is close to a threshold.

We have illustrated in Fig. 5 that for a warming cli- 
mate system, the variability caused by the freshwater forcing may increase. A similar effect is obtained by applying a larger amplitude of the stochastic freshwater forcing. But it is evident from Fig. 6a that the probability for a THC collapse increases toward larger forcing amplitudes. We thus propose that the following positive feedback mechanism operates close to the instability threshold: global warming potentially weakens the NADW formation directly by warming and freshening the North Atlantic surface water, thereby destabilizing the THC and leading to a certain probability for a collapse. The sensitivity of the destabilized THC to stochastic processes (e.g., weather) will therefore increase, causing larger variability in the strength of the THC. This further increases the probability for a collapse, since it is more likely for the THC to cross the critical threshold when the fluctuations around the mean state are larger.

\section{Conclusions}

By running ensemble simulations, we have shown that the ocean thermohaline circulation shares many characteristics with nonlinear systems. These become particularly evident when the model approaches a stability threshold. However, in none of the cases does the model appear to be chaotic. The dynamics of the model are rather restricted by the fact that the applied freshwater perturbations are independent of the model state and are simply prescribed through a random number sequence, by the simplicity of the atmospheric model component, the simple parameterizations of many processes, and by the reduced dimensions. As pointed out by Palmer (2001), the use of bulk parameterizations for unresolved processes may lead to significant errors in model simulations, even if the unresolved scales describe only a small fraction of the total variance of the system. On the other hand, neglecting processes tends to reduce, rather than increase, the degrees of freedom of the system. We therefore expect that our simplified model shows only a part of the possible response types of the earth system, and that at least the qualitative mechanisms presented here are robust.

While the strength of the THC has been relatively stable during the Holocene, future warming may push it toward an instability threshold, and possibly beyond it. Our results indicate that for a climate sensitivity range of about $0.5^{\circ} \mathrm{C}$ around this threshold, processes that are not resolved in the model may cause enough variability to make projections regarding the fate of the THC difficult or even impossible. The simulations also suggest that even if we know that the THC will approach but not reach the instability threshold, the predictability of the future THC strength is severely limited. The current knowledge about possible instability thresholds in the natural climate system is therefore not sufficient. We need to know the current position of the climate system in phase space, and how close the system will move to
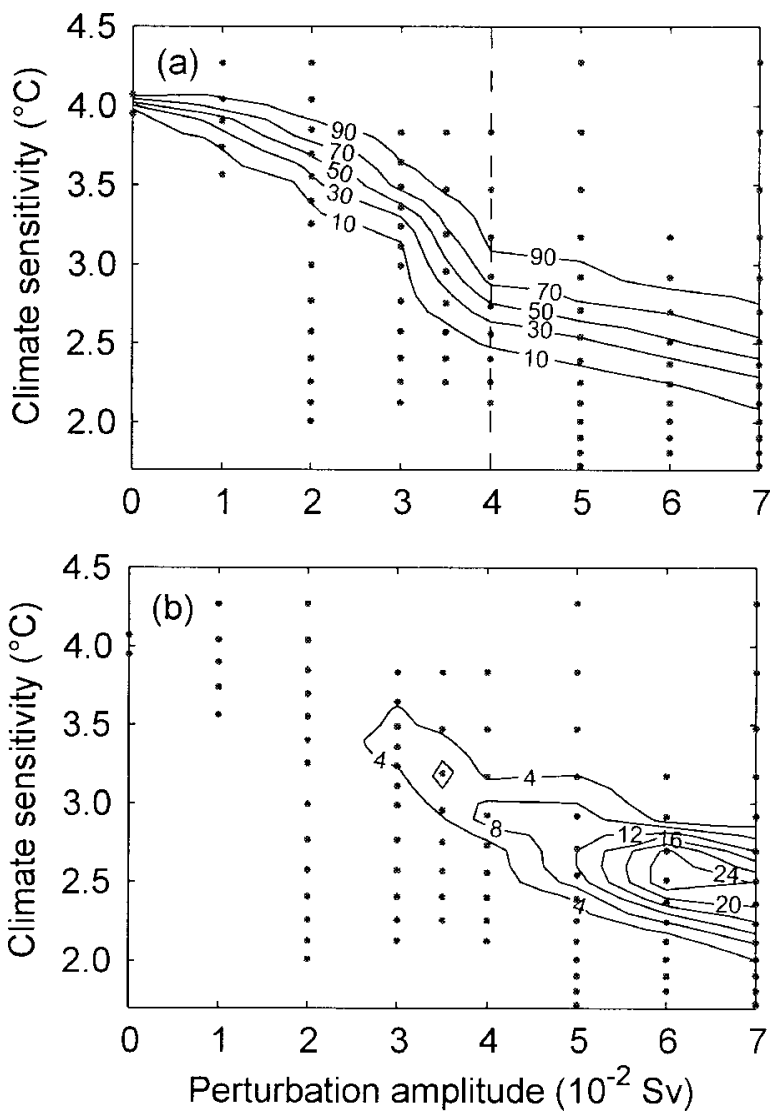

FIG. 6. (a) Probability (\%) for the THC being collapsed in year 1000 after the warming vs stochastic forcing and climate sensitivity. Oscillations in the THC strength occur only above a perturbation amplitude of 0.04 Sv, marked by the vertical dashed line. (b) Probability for the THC collapsing between year 1000 and 2000 after the warming. (b) Indicates that there may be a substantial chance for a THC shutdown occurring long after the warming has stopped.

a threshold. The use of more complex and comprehensive climate models will certainly improve future climate projections by a more accurate representation of the processes operating in the climate system. But reliable predictions of the future climate will also require a large number of ensemble simulations to assess the uncertainty of the model projections as well as longterm runs extending over many centuries before conclusions about the fate of the thermohaline circulation can be drawn. This is a computationally expensive task to carry out, but evidence from models of different complexity that the THC may not be far away from an instability threshold (Walin 1985; Tziperman et al. 1994; Tziperman 2000), and the fact that we are probably approaching it by man-made greenhouse warming (Houghton et al. 2001), should be of sufficient concern to intensify research into this topic.

Acknowledgments. This work was supported by the Swiss National Science Foundation and the EC Project Milleclim. We enjoyed discussions with K. Plattner, J. 
Hirschi, and J. Flückiger. Comments by E. Tziperman and an anonymous reviewer are acknowledged.

\section{REFERENCES}

Aeberhardt, M., M. Blatter, and T. F. Stocker, 2000: Variability on the century time scale and regime changes in a stochastically forced, zonally-averaged ocean-atmosphere model. Geophys. Res. Lett., 27, 1303-1306.

Delworth, T. L., and K. W. Dixon, 2000: Implications of the recent trend in the Arctic/North Atlantic oscillation for the North Atlantic thermohaline circulation. J. Climate, 13, 3721-3727.

Frankignoul, C., and K. Hasselmann, 1977: Stochastic climate models. Part II: Application to sea-surface temperature anomalies and thermocline variability. Tellus, 29, 289-305.

Gent, P. R., 2001: Will the North Atlantic Ocean thermohaline circulation weaken during the 21 st century? Geophys. Res. Lett. 28, 1023-1026.

Hall, A., and R. J. Stouffer, 2001: An abrupt climate event in a coupled ocean-atmosphere simulation without external forcing. Nature, 409, 171-174

Hasselmann, K., 1976: Stochastic climate models. Part I: Theory. Tellus, 28, 473-485.

Houghton, J. T., Y. Ding, D. J. Griggs, M. Noguer, P. J. van der Linden, X. Dai, K. Maskell, and C. A. Johnson, Eds., 2001: Climate Change 2001: The Scientific Basis. Contribution of Working Group I to the Third Assessment Report of the Intergovernmental Panel on Climate Change. Cambridge University Press, $881 \mathrm{pp}$.

Joos, F., G.-K. Plattner, T. F. Stocker, O. Marchal, and A. Schmittner, 1999: Global warming and marine carbon cycle feedbacks on future atmospheric $\mathrm{CO}_{2}$. Science, 284, 464-467.

Knutti, R., and T. F. Stocker, 2000: Influence of the thermohaline circulation on projected sea level rise. J. Climate, 13, 19972001

- - , and D. G. Wright, 2000: The effects of subgrid-scale parameterizations in a zonally averaged ocean model. J. Phys. Oceanogr. 30, 2738-2752.

Latif, M., E. Roeckner, U. Mikolajewicz, and R. Voss, 2000: Tropical stabilization of the thermohaline circulation in a greenhouse warming simulation. J. Climate, 13, 1809-1813.

Manabe, S., and R. J. Stouffer, 1988: Two stable equilibria of a coupled ocean-atmosphere model. J. Climate, 1, 841-866. , and — 1993: Century-scale effects of increased atmospheric $\mathrm{CO}_{2}$ on the ocean-atmosphere system. Nature, 364, 215-218.
Mikolajewicz, U., and E. Maier-Reimer, 1994: Mixed boundary conditions in ocean general circulation models and their influence on the stability of the model's conveyor belt. J. Geophys. Res., 99, 22 633-22 644.

Palmer, T. N., 1999: A nonlinear dynamical perspective on climate prediction. J. Climate, 12, 575-591.

_ 2001: A nonlinear dynamical perspective on model error: A proposal for nonlocal stochastic-dynamic parameterization in weather and climate prediction models. Quart. J. Roy. Meteor. Soc., 127, 279-304.

Rahmstorf, S., 1995: Bifurcations of the Atlantic thermohaline circulation in response to changes in the hydrological cycle. Nature, 378, 145-149.

Schmittner, A., and T. F. Stocker, 1999: The stability of the thermohaline circulation in global warming experiments. J. Climate, 12, 1117-1133

—, and _ 2001: A seasonally forced ocean-atmosphere model for paleoclimate studies. J. Climate, 14, 1055-1068.

Stocker, T. F., and D. G. Wright, 1991a: Rapid transitions of the ocean's deep circulation induced by changes in surface water fluxes. Nature, 351, 729-732.

_ haline circulation. Part II: Interocean exchanges in the PacificAtlantic basin system. J. Phys. Oceanogr., 21, 1725-1739.

- and A. Schmittner, 1997: Influence of $\mathrm{CO}_{2}$ emission rates on the stability of the thermohaline circulation. Nature, $\mathbf{3 8 8}, 862-$ 865

— D. G. Wright, and L. A. Mysak, 1992: A zonally averaged, coupled ocean-atmosphere model for paleoclimate studies. $J$. Climate, 5, 773-797.

Stommel, H., 1961: Thermohaline convection with two stable regimes of flow. Tellus, 13, 224-241.

Stott, P. A., S. F. B. Tett, G. S. Jones, M. R. Allen, J. F. B. Mitchell, and G. J. Jenkins, 2000: External control of 20th century temperature by natural and anthropogenic forcing. Science, 290, 2133-2137.

Tziperman, E., 1997: Inherently unstable climate behavior due to weak thermohaline ocean circulation. Nature, 386, 592-595.

- 2000: Proximity of the present-day thermohaline circulation to an instability threshold. J. Phys. Oceanogr., 30, 90-104.

—, R. J. Toggweiler, Y. Feliks, and K. Bryan, 1994: Instability of the thermohaline circulation with respect to mixed boundary conditions: Is it really a problem for realistic models? J. Phys. Oceanogr., 24, 217-232.

Walin, G., 1985: The thermohaline circulation and the control of ice ages. Paleogeogr. Paleoclim. Paleoecol., 50, 323-332. 